\title{
News Items
}

Nachrichten - Nouvelles - Noticias - Notizie

Nota Necrologica relativa al Dr. K. Alcalá-Santaella

La muerte inesperada del Profesor R. Alcalá-Santaella en Valencia (España), después de una brevisima y fulminante enfermedad, en Octubre de 1959, ha sido muy sentida por todos sus compañeros y amigos, y de estos últimos tenía muchis por su extraordinaria simpatía y exquisita corrección.

Era una figura muy conocida en los medios urológicos, especialmente en los Con-gresos de la Sociedad Internacional de Urología.

Comenzó en 1921 sus estudios de Urología con el Prof. D. Leonardo de la Peña. Fué pensionado por la Junta de Ampliación de Estudios en 1922 para trabajar en el Hospital Necker, al lado del Prof. Legueu. Asístió también a la Clínica del Prof. Marion, en Paris.

Su título de Doctor fué obtenido con la calificación de Sobresalíente, con un trabajo sobre «Los vasos y conductos excretores del riñón» (año 1923).

Parejamente a su interés por la Urología, Alcalá-Santaella tenía tan sólida formación anatómica y tan gran vocación por esta rama científica, que obtuvo en 1930 la Cátedra de Anatomía

Descriptiva y Topográfica de la Facultad de Medicina de Cadiz. Aquel mismo año de 1930 fué otra vez pensionado, realizando estudios especiales en Munich y en Viena. En 1935 consiguió la Cátedra de Anatomía de Valencia, donde ha residido hasta su muerte.

En 1941 fué nombrado Profesor Agregado de Urología de la Facultad de Valencia, y desde este año dirigía también el Servicio de Urología del Hospital Provincial de aquella ciudad.

La lista de sus publicaciones es especialmente larga y contiene 149 títulos. Entre ellos se destacan sus primeras publicaciones anatórnicas sobre vascularización renal, y su obra de Urología Clínica, publicada en varios fascículos en 1942, 1943 y 1944.

Descanse en paz nuestro buen amigo.

Luis Cifuentes Delatte

First International Congress of Nephrology

Geneva, Switzerland, September 1, 1960, and Evian (Haute-Savoie), France, September 2 to 4, 1960

organised by La Société de Néphrologie, The Scandinavian Society for Kidney

Research, The American Society for Clinical Investigation, Società Italiana di

Nefrologia and The Renal Association.

Scientific Programme

Thursday, September 1, 1960 (at Geneva):

"Regulation of Tubular Ionic Exchange". Reports given by R. W. Berliner, Bethesda, Md., F. Morel, Paris, S. W. Stanbury, Manchester, F. C. Bartter, Be-thesda, Md., A. F. Muller, Geneva, G. Fanconi, Zurich.

Friday, September 2 (at Evian): Morning: 
Round Table Discussions: "Glomerular Ultrastructure" and "Stop Flow Technics". Plenary Session: "Kidney Transplantation in Man" (Conference by J. Hamburger, Paris, and J. P. Merrill, Boston, Mass.).

328 News Items - Nachrichten - Nouvelles - Noticias - NotizicX/1960

Afternoon:

"Shock Kidney". Reports given by P. Teschan (U.S. Army), A. П. Mason (U.S. Army), G.

Richet, Paris, P. Vassali, Paris, L. Micone, Parma, M. Derot, Paris, J. L. Funck-Brentano, Paris, J. P. Merrill, Boston, Mass.

Saturday, September 3 (at Evian): Round Table Discussion: "Diuretics".

Plenary Session: "Renal Acidosis" (Conference by W. B. Schwartz, Boston,

Mass.).

Sunday, September 4, 1960 (at Evian):

"Recent Advances in Nephrology". Chairman: F. C. Reubi, Berne.

(Mailing address: Professor G. Richet, M.D., Hôpital Necker, 149, rue de Sevres, Paris XVe

(France).

VI Cougreso Argentine de Urologia

Rosario, 22, 23, 24 y 25 de Septiembre de I960

Tema oficial: «Hidronefrosis» (Mauricio Firstater, Cordoba, y Frank Hughes, Montevideo, relatores).

Mesas redondas:

«Tumores Parrarrenales»;

«lnduración Plástica de los Cuerpos Cavernosos».

3. Temas libres.

(Rolando Herenu, secretario, Círculo Medico de Rosario, Italia 673, Rosario, República

Argentina.)

Association Française d'Urologie

$1^{\circ} 54 \mathrm{e}$ Congrès, Paris, Septembre 1960

Rapport: «Traitement des méga-uretères» (J. Perrin, I.yon, rapporteur).

Forum: «Induration plastique des corps cavernezX».

$2^{\circ}$ 55e Congrès, Paris, 1961

Rapport: «Des hypertensions artérielles justiciables de la néphrectomíe» (J. Michon, Paris, rapporteur).

(Pierre Delinotte, secretaire general, 3, rue Delaizement, Paris 17e, France.)

$\mathrm{XXXIII}^{\circ}$ Congresso della Società Italiana di Urologia

Bergamo e Brescia, Seconda Metà delГOttobre 1960

$1^{\circ}$ Tema di Relazione: «lpertensione arteriosa di origine renale di interesse chírurgico» (Vittorio Pettinari, relatore, Padova).

$2^{\circ}$ Simposíum: «Ureteriti e periureteriti» (Giuseppe Nisio, moderatore, Barí). (Alberto

Oberholtzer, segretario, Viale Angelico 90, Roma, Italia.) 